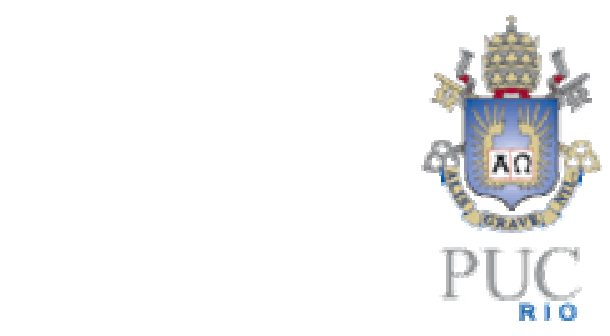

Mariana Gava Reddo Alves

\title{
O Efeito Coorte na Preferência da Aparência Física
}

Dissertação de Mestrado

Dissertação apresentada como requisito parcial para obtenção do título de Mestre pelo Programa de PósGraduação em Administração da PUC-Rio.

Orientador: Paulo Cesar Motta

Rio de Janeiro, agosto de 2006 


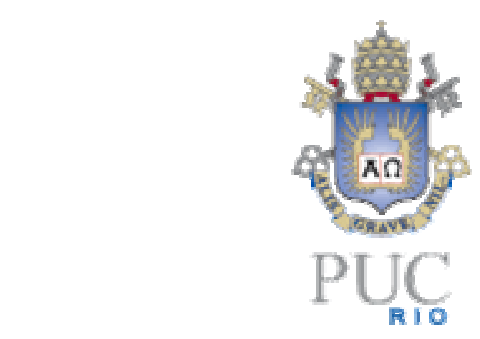

Mariana Gava Reddo Alves

\title{
O Efeito Coorte na Preferência da Aparência Física
}

\author{
Dissertação apresentada como requisito parcial para obtenção \\ do título de Mestre pelo Programa de Pós-Graduação em \\ Administração da PUC-Rio. Aprovada pela Comissão \\ Examinadora abaixo assinada.
}

\author{
Paulo Cesar Motta \\ Orientador \\ PUC-Rio \\ Paulo Cesar Motta \\ PUC-Rio \\ Patrícia Amélia Tomei \\ PUC-Rio \\ Mônica Zaidan Gomes Rossi \\ FACC/UFRJ \\ João Pontes Nogueira
}

Coordenador(a) Setorial do Centro de Ciências Sociais - PUC-Rio

Rio de Janeiro, 31 de agosto de 2006 
Todos os direitos reservados. É proibida a reprodução total ou parcial do trabalho sem autorização da universidade, da autora e do orientador.

\section{Mariana Gava Reddo Alves}

É Mestre em Administração de Empresas pela PUC-Rio. Especialista, com M.B.A em Marketing pela Fundação Getúlio Vargas. Bacharel em Comunicação Social, com habilitação em Publicidade e Propaganda pela Universidade Federal do Rio de Janeiro (U.F.R.J). Participou de diversos congressos de integração latinoamericanos na área de administração de empresas. Profissionalmente exerceu cargos de coordenação e gerência na área de Merchandising no setor varejista. Atualmente atua como Gerente de Merchandising do Ponto Frio.

Ficha Catalográfica

Alves, Mariana Gava Reddo

O Efeito-Coorte na Preferência da Aparência Física / Mariana Gava Reddo Alves ; orientador Paulo César Motta. - Rio de Janeiro : PUC, Departamento de Administração, 2006.

118 f. : il. ; $30 \mathrm{~cm}$

Dissertação (mestrado) - Pontifícia Universidade Católica do Rio de Janeiro, Departamento de Administração.

Incluí referências bibliográficas.

1. Administração - Teses; 2. Marketing; 3. Coorte; 4. Aparência física; 5 . Comportamento do consumidor; 6 . Preferência; 7. Período. I. Motta, Paulo Cesar. II. Pontifícia Universidade Católica do Rio de Janeiro. Departamento de Administração. III. Título. 
Este trabalho é dedicado à minha mãe, meu grande incentivo e inspiração para a realização deste curso, ao meu pai, meu suporte e porto seguro em todas as horas, e ao meu irmão, minha alegria e minha criatividade. 


\section{Agradecimentos}

Ao meu orientador Paulo César Motta pela orientação sempre presente e persistente, fundamental à realização deste trabalho.

A todos os professores e funcionários do Departamento de Administração pela excelência dos serviços prestados e por terem tornado essa experiência ainda mais gratificante.

Às amigas Ana Raquel Rocha e Patrícia Grossi, pela força, incentivo e companhia mesmo nos momentos mais solitários, e pela amizade que cresceu e fortaleceu ao longo do curso.

A todos os amigos que fiz durante essa caminhada.

A todos aqueles que de uma forma ou de outra me ajudaram aplicando questionários ou mesmo só respondendo, principalmente a ajuda dos Amigos Gisele Andrade, Cristiana Velasco, Raquel Scrivano, Seiva Emanuel, Carina Guedes, Bianca da Hora, Juliana Schramm, Beatrice Neves, Lavinia Frota, Ana Carolina Gava e, como não podia deixar de ser, minha mãe. 


\section{Resumo}

Alves, Mariana Gava Reddo. O Efeito Coorte na Preferência da Aparência Física. Rio de Janeiro, 2006. 118p. Dissertação de Mestrado Departamento de Administração, Pontifícia Universidade Católica do Rio de Janeiro.

Esta pesquisa investiga a correlação entre a idade das pessoas e suas preferências pela aparência física, que possa vir a ajudar no entendimento do consumo de produtos influenciados pela estética e identificar que tipo físico seria mais indicado para campanhas de marketing. Investiga, particularmente, como a associação da idade com a preferência pode ser explicada por meio do efeitocoorte. Diversos estudos mostram a relevância do efeito coorte no estabelecimento da preferência por diversos produtos e serviços. O estudo levanta hipóteses que foram testadas a partir de dados levantados por meio de questionário em que atores e atrizes indicados para o Oscar desde a década de quarenta que são avaliados segundo critérios de aparência física. Os resultados mostram que, em geral, existe um período crítico de idade em acontece a formação das preferências. Os resultados realçam diferenças marcantes entre as respostas quando classificadas pelo gênero do respondente.

\section{Palavras-chave}

1. Administração - Teses; 2. Marketing; 3. Coorte; 4. Aparência física; 5. Comportamento do consumidor; 6. Preferência; 7. Período 


\section{Abstract}

Alves, Mariana Gava Reddo. The Cohort Effect on Personal Appearance. Rio de Janeiro, 2006. 118p. MSc. Dissertation Departamento de Administração, Pontifícia Universidade Católica do Rio de Janeiro.

This research investigates the correlation between peoples ages and their preference for certain physical types, seeking to understand how such preferences may influence the consumption of aesthetics-related products and thus, helping to determine which physical traits would be the most appropriate in marketing campaigns. It particularly investigates in which ways the association between age and preference can be explained by means of the cohort effect. Many studies have shown the relevance of the cohort effect in the establishment of consumer preference for certain products and services. This study identifies hypothesis that were tested based on data gathered from questionnaires in which actors and actresses who were Oscar-nominees since the 1940's were evaluated according to physical appearance criteria. The results show that, generally, there is a critical period of age during which peoples preferences are formed. The results also highlight the significant differences amongst the answers when respondents are classified according to gender.

\section{Keywords}

1. Administration - Thesis; 2. Marketing; 3. Cohort; 4. Personal Appearance; 5. Consumer Behavior; 6. Preference; 7. Period. 


\section{Sumário}

1 O PROBLEMA 13

1.1. Introdução 13

1.2. Objetivo 14

1.3. Relevância do Estudo 15

1.4. Delimitação do Estudo 16

2 REFERENCIAL TEÓRICO

2.1. Aparência 17

2.2. O Efeito Coorte 20

2.3. O Fenômeno do Período Crítico 26

2.4. Efeito Idade, Efeito Período e Nostalgia 27

2.5. Hipóteses 28

3 METODOLOGIA 30

3.1. A Pesquisa 30

3.2. A Elaboração do Questionário 31

3.2.1. A Seleção dos Artistas 31

3.2.2. O Enunciado das Questões $\quad 37$

3.3. Amostra 39

3.4. Coleta de Dados 42

3.5. Tratamento dos Dados 43

3.5.1. Normalização das Respostas 44

3.5.2. Variável Latente 44

3.5.3. Método Estatístico de Análise 47

3.6. Limitações do Método 48

4 RESULTADOS $\quad 50$

4.1. Existência de um período crítico na preferência da aparência física 50

4.2. Avaliação da aparência física na perspectiva feminina e masculina 52

4.2.1. Avaliação Masculina $\quad 53$

4.2.2. Avaliação Feminina $\quad 55$

4.3. Avaliações do Sexo Oposto x Mesmo Sexo 57 
4.3.1. Homens avaliando Atores 58

4.3.2. Homens avaliando Atrizes 62

4.3.3. Mulheres avaliando Atores 65

4.3.4. Mulheres avaliando Atrizes $\quad 67$

4.4. Demais resultados $\quad 71$

4.4.1. Homens x Ator Talentoso 71

4.4.2. Homem x Atriz Talentosa 72

4.4.3. Mulher $x$ Ator Talentoso 73

4.4.4. Mulher x Atriz Talentosa 74

5 DISCUSSÃO E CONCLUSÃO

5.1. Avaliação Geral da Amostra 76

5.2. Avaliação Masculina

$\begin{array}{ll}\text { 5.3. Avaliação das Mulheres } & 78\end{array}$

5.4. A segunda parte do questionário $\quad 80$

5.4.1. A Avaliação Masculina 80

5.4.2. A Avaliação Feminina $\quad 81$

5.5. Limitações dos Resultados

5.6. Sugestões para Estudos Futuros

6 REFERÊNCIAS BIBLIOGRÁFICAS

7 ANEXOS 94

7.1. Anexo $1 \quad 94$

7.2. Anexo 2 - Questionário 104 


\section{Lista de figuras}

Figura 1: Amadurecimento, sucessão de coortes e diferenças de idade

(Fonte:Riley, 1973).

22

Figura 2: Variação do SSA 


\section{Lista de Gráficos}

Gráfico 1: Distribuição de freqüência absoluta da amostra por idade e sexo. 40

Gráfico 3: Distribuição das médias normalizadas por SSA 51

Gráfico 4: Distribuição das notas dos Homens por SSA 54

Gráfico 5: Distribuição das notas das mulheres por SSA 56

Gráfico 6: Distribuição das notas de Homens avaliando atores por SSA. $\quad 60$

Gráfico 7: SSA dos homens ao avaliarem o ator que mais lhe chamou a atenção pela aparência física. $\quad 60$

Gráfico 8: SSA dos homens ao avaliarem o ator que gostariam de ser. $\quad 61$

Gráfico 9: Distribuição das notas de Homens avaliando Atrizes por SSA. 63

Gráfico 10: SSA dos homens ao avaliarem a atriz que mais the chamou a atenção pela aparência física. $\quad 64$

Gráfico 11: Distribuição das notas das Mulheres avaliando Atores por SSA. 66

Gráfico 12: SSA das mulheres ao avaliarem o ator que mais lhe chamou a atenção pela aparência física. 66

Gráfico 13: Distribuição das notas das Mulheres avaliando Atrizes por SSA. 69

Gráfico 14: SSA das mulheres na avaliação da atriz que mais the chamou a atenção pela aparência física. $\quad 69$

Gráfico 15: SSA das mulheres na avaliação da atriz que gostariam de ser. $\quad 70$

Gráfico 16: SSA dos homens na avaliação do ator que consideravam mais talentoso. $\quad 72$

Gráfico 17: SSA dos homens na avaliação da atriz que consideravam mais talentosa.

Gráfico 18: SSA das mulheres na avaliação do ator que consideravam mais talentoso.

Gráfico 19: SSA das mulheres na avaliação da atriz que consideravam mais talentosa. 


\section{Lista de tabelas}

Tabela 1: Grupos de coorte norte-americanos (Fonte: Meredith e Schewe, 2002).

Tabela 2: Grupos de coorte brasileiros (Fonte: Motta, Rossi e Schewe, 2002). 25

Tabela 3: Grupos formados para representar um período de sucesso. 33

Tabela 4: Lista Final dos Atores Selecionados. 35

Tabela 5: Lista Final das Atrizes Selecionadas. 37

Tabela 6: Distribuição da freqüência acumulada da amostra por idade e sexo. 42

Tabela 7: Exemplo de normalização das notas 44

Tabela 8: Exemplo de SSA 45

Tabela 9: Notas normalizadas por SSA 46

Tabela 10: Exemplo de média das notas por SSA. 46

Tabela 11: Resultados da regressão das notas dos artistas por SSA 52

Tabela 12: Resultados da regressão das notas dos Homens por SSA 54

Tabela 13: Resultados da regressão das notas das Mulheres por SSA 56

Tabela 14: Resultados da regressão da média das notas dos Homens avaliando Atores

61

Tabela 15: Resultados da regressão da média das notas dos Homens avaliando Atrizes

Tabela 16: Resultados da regressão da média das notas das Mulheres avaliando Atores.

Tabela 17: Resultados da regressão das notas das Mulheres avaliando

Atrizes.

Tabela 18: Comparação dos resultados obtidos com a literatura existente. $\quad 80$ 